\title{
De la "Ciudad del Acero" al "Desarrollo Local". Propuestas para una aproximación socioantropológica a las relaciones entre industria y ciudad en el caso de San Nicolás de los Arroyos
}

\author{
Julia Soul \\ Centro de Estudios e Investigaciones Laborales \\ (CEIL) - CONICET \\ juliasoul@hotmail.com
}

\begin{abstract}
Resumen
En este trabajo, proponemos construir una mirada de mediano plazo sobre la dinámica de las relaciones entre la ciudad de San Nicolás de los Arroyos y la planta siderúrgica instalada allí en la década '50, gestionada y dirigida durante treinta años por el Estado Nacional, y privatizada en 1992. Específicamente, interesa indagar la forma en que un conjunto de prácticas e intervenciones referidas a los procesos de producción y reproducción de la fuerza de trabajo concretizan el proceso de formación de los obreros industriales como parte de la clase trabajadora en el seno de procesos hegemónicos consecutivos. En segundo lugar, interesa trazar coordenadas analíticas y problemáticas para un abordaje socioantropológico de las relaciones entre empresa y comunidad de emplazamiento. En este trabajo, procuramos recuperar las especificidades históricas de la relación SOMISA - San Nicolás de los Arroyos así como establecer líneas de análisis de la situación contemporánea, con un punto de tracción común: los entramados relacionales y de prácticas que arrojan luz sobre el accionar obrero respecto de los principales problemas detectados para cada momento: vivienda y condiciones de habitabilidad desde la década del '60 y problemas de empleo para la década del ' 90.
\end{abstract}

Palabras Clave: Industria - Comunidad - Procesos Hegemónicos - Clase Obrera

\begin{abstract}
This paper proposes a medium term approach to relationships between the city of San Nicolás de los Arroyos and the steel mill installed there by the 50's, managed and directed for thirty years by the national government, and privatized in 1992. Specifically, we are interested in investigating how a set of practices and interventions relating to the processes of production and reproduction of the labor force concretize the formation of industrial workers as part of the working class within consecutive hegemonic processes. Second, we are interest in tracing analytical coordinates for a socio-anthropological approach to the relationship between industry and community site. In this paper, we try to recover the historical specificities of the relationship SOMISA - San Nicolas de los Arroyos and to establish lines of analysis of the contemporary situation with a common coupling point: the relational and practical frameworks
\end{abstract}

SOUL, María Julia, "De la ‘Ciudad del Acero' al 'Desarrollo Local'. Propuestas para una aproximación socioantropológica a las relaciones entre industria y ciudad en el caso de San Nicolás de los Arroyos", en Avances del Cesor, Año X, N 10, 2013, pp. 173-196. 
that shed light on the workers' actions on the main issues identified for each time: housing and living conditions since the 60 s and employment issues for the 90 s.

Key Words: Industry - Community - Hegemonic Processes - Working Class

\section{Introducción ${ }^{1}$}

En este trabajo, proponemos construir una mirada de mediano plazo sobre la dinámica de las relaciones entre la ciudad de San Nicolás de los Arroyos y la planta siderúrgica instalada allí en la década '50, gestionada y dirigida durante treinta años por el Estado Nacional, y privatizada en 1992. El objetivo es recuperar los procesos mediante las cuales diferentes conjuntos sociales configurados en torno de la instalación de una gran industria fueron articulando prácticas, instituciones e intervenciones que se desplegaron contradictoriamente y contribuyeron a modelar la ciudad. Específicamente, interesa indagar la forma en que este conjunto de prácticas e intervenciones - que remiten a los procesos de producción y reproducción de la fuerza de trabajo- concretiza el proceso de formación de los obreros industriales como parte de la clase trabajadora en el seno de procesos hegemónicos consecutivos.

En segundo lugar, interesa trazar coordenadas analíticas y problemáticas para un abordaje socioantropológico de las relaciones entre empresa y comunidad de emplazamiento. Esta relación ha sido objeto de numerosas líneas de investigación en diversos campos disciplinares. En el contexto de la sociología y la antropología, se demarcaron dos grandes áreas problemáticas. De una parte, las investigaciones acerca de la forma company town que atendieron a la configuración de los mercados de trabajo en regiones 'vacías" ${ }^{2}$-especialmente

1 Las líneas de investigación en las que se inscribe este artículo se desarrollan en los proyectos dirigidos por la Dra. Claudia Figari con asiento en el CEIL: PIP "Prácticas hegemónicas corporativas y disciplinamiento laboral: saberes, prácticas y posiciones de los trabajadores en contextos de racionalización y fragmentación en el trabajo" (2010-2012) y PICT "Hegemonía empresarial y estrategias de disciplinamiento laboral: disputas dentro y fuera de las fábricas “(2011-2013) y en el PID "Industria, ciudad y relaciones obrero - empresarias en comunidades urbanas” dirigido por la Dra. Silvia Simonassi con asiento en el ISHIR - CESOR.

2 SIERRA ALVAREZ, José, El Obrero Soñado. Ensayos sobre el paternalismo industrial. Editorial Siglo XXI. Barcelona. 1990; NEIBURG, Federico, Fábrica y Villa Obrera. Historia Social y Antropología de los Obreros del cemento, Centro Editor de América Latina, Buenos Aires, 1989; LEITE LOPES, José Sergio,"Fabrica e vila operaria. Consideraçoes sobre uma forma de servidão burguesa”, en AAVV Mudanza social no Nordeste, Ed. Paz e Terra. Río de Janeiro, 1979 y A Tecelagem dos Conflitos de Classe Na Cidade das Chaminés, Marco Zero Brasilia, 1988; MORAES MOREL, Regina, A ferro e fogo Construção e crise da família siderúrgica: o caso de Volta Redonda, Tese de Doutoramento, Sao Paulo, Brasil, 1989. LUPANO, María M., La gran familia industrial: espacio urbano, prácticas sociales e ideología (1870 - 1945), Buenos Aires, Santiago Arcos, 2009. 
en el caso de industrias extractivas o agroindustrias- de modo coherente con la necesidad de configuración de subjetividades disciplinadas al trabajo fabril y de colectivos obreros particulares. ${ }^{3}$ Estudios recientes indagan la extensión de la forma company town como modo predominante de establecimiento de las grandes industrias en el continente. ${ }^{4}$ Por otra parte, las transformaciones que operan en las ciudades a partir de las relaciones de poder que dinamizan la instalación de grandes empresas. ${ }^{5}$ El vector de estas investigaciones remite a las particulares configuraciones de las relaciones de dominación capital - trabajo en estos espacios sociales, que presentarían una serie de características que contestarían parcialmente a la relación asalariada típica de los contextos de gran industria desarrollada por Marx en $E l$ Capital. Las relaciones personalizadas en contraposición a las anónimas y la presencia de la empresa en el ámbito de la reproducción de la fuerza de trabajo que limitaría el carácter 'libre' de la fuerza de trabajo son los principales elementos que determinarían la configuración de grupos obreros con prácticas organizativas y políticas particulares. Asimismo, la mayor parte de las investigaciones se sitúan en un momento histórico en el que los procesos de expansión del capital operan a través de formas estatales que no han desarrollado en su totalidad las funciones hegemónicas y que, por lo tanto, dejan librada a la iniciativa privada las intervenciones relativas a la reproducción de la clase obrera. ${ }^{6}$ Las investigaciones en esta clave, dejan constancia de los procesos de transformación de las relaciones en los company towns a partir de procesos de conflictividad y de transformaciones en los procesos hegemónicos. Sin embargo, permanece abierta la pregunta acerca de la continuidad de las relaciones entre empresa y territorio de emplazamiento una vez que finaliza el dominio monopólico de la primera sobre el segundo.

En este trabajo, procuramos recuperar las especificidades históricas de la relación SOMISA $^{7}$ - San Nicolás de los Arroyos así como establecer líneas de análisis de la situación contemporánea, con un punto de tracción común: los entramados relacionales y de prácticas que arrojan luz sobre el accionar obrero respecto de los principales problemas detectados

3 MOSHER, Anne, "Something better than the best": Industrial Restructuring, George McMurtry and the Creation of the Model Industrial Town of Vandergrift, Pennsylvania, 1883-1901 (1995)", en Annals of the Association of American Geographers, March 1995, V. 85, № 1.

4 DINIUS Oliver y VERGARA Ángela, Company towns in the Americas. Athens Press, Chicago, 2010.

5 NASH June, From tank town to high tech. the clash of community and industrial cycles. Suny Press, Nueva York, 1989; ARENSBERG, Conrad, "Industry and Community" en American Journal of Sociology, 1942, № 48.

6 Este es uno de los principales aportes conceptuales de SIERRA ALVAREZ, José, El Obrero...,Op. Cit. La relación con el Estado también está contenida en la categoría Sistema de Fábrica con Villa Obrera, ver LEITE LOPES José Sergio,"Fabrica e vila...”, Op. Cit.

7 Sociedad Mixta Siderúrgica Argentina. 
para cada momento: vivienda y condiciones de habitabilidad desde la década del '60 y problemas de empleo para la década del '90. Proponemos una aproximación de mediano plazo que permita considerar continuidades y rupturas entre procesos hegemónicos consecutivos. La hipótesis de trabajo es que el entramado relacional configurado durante el nacionalismo industrialista ${ }^{8}$ se expresa en un colectivo fabril cuyas segmentaciones jerarquizadas se proyectan a la ciudad, y se traducen en condiciones de vida y reproducción diferenciadas. En torno de estas últimas, los trabajadores estructuraron un conjunto de prácticas e instituciones mediante las cuales procuraron la obtención de sus demandas y objetivos. Este entramado institucional transforma de manera sustancial sus funciones y su dinámica con la crisis que provocó el advenimiento del proceso hegemónico neoliberal, que en San Nicolás no significó la pérdida de centralidad de la gran empresa como principal actor económico regional. Estas transformaciones se producen en virtud de modificaciones en la forma de Estado y en las intervenciones empresarias respecto del colectivo de trabajo y de la comunidad toda. Consideramos que la aproximación a la esta relación en un caso singular y en el mediano plazo, puede contribuir a la construcción de explicaciones en torno del accionar de fracciones de la clase obrera industrial, mediante la incorporación en el análisis de prácticas vinculadas con las condiciones de reproducción.

\section{La instalación de SOMISA. Forma de company town y segmentación de la fuerza de trabajo}

Desde la década del '40 se profundizó el proceso de industrialización sustitutiva en la región de San Nicolás de los Arroyos, cuando -además del impulso que tomaban la industria alimentaria, la textil y la metalúrgica- se localizan allí inversiones industriales decididas en forma centralizada por el Estado Nacional. Hasta ese momento, la ciudad del Acuerdo era una ciudad típica de la Pampa húmeda e integrada desde finales del siglo XVIII al capitalismo agrario mediante el cual el país se insertó en la división internacional del trabajo. ${ }^{9}$ El hito más

8 En nuestra tesis doctoral, hemos denominado nacionalismo industrialista al proceso hegemónico sustentado en el proceso de acumulación de industrialización sustitutiva, en el seno del cual la actividad industrial adquiría centralidad para el progreso de la nación. En este contexto es que la industria siderúrgica adquiere valor estratégico como vector de lo nacional, asociada a nociones de progreso, bienestar y desarrollo. Para las apropiaciones y resignificaciones que los diferentes actores sociales ver SOUL, Julia, Relaciones de clase y construcción de una comunidad de fábrica en la ex SOMISA. Tesis de Doctorado en Humanidades mención Antropología, UNR, Rosario, 2010, p. 29 ss (mimeo).

9 Durante la primera mitad del siglo XX, pequeños establecimientos industriales elaboraban productos alimenticios y de consumo doméstico y el emblema industrial de la zona era la fábrica textil situada en La Emilia, típico establecimiento industrial con importante incidencia en el ámbito de la reproducción de la fuerza de trabajo, que cerró sus puertas hacia la década del 70. PRIMO, Ricardo, 
importante del proceso de industrialización es la instalación de planta siderúrgica integrada de SOMISA, que para la década del ' 60 se había convertido en el referente simbólico de la ciudad, fundante del pasaje de Ciudad del Acuerdo a la Ciudad del Acero.

Desde los inicios de la construcción de la planta siderúrgica, en la década del '50, la ciudad se convirtió en un polo de atracción de trabajadores rurales, provenientes de familias de pequeños campesinos o campesinos proletarizados, que llegaban desde áreas económicas marginadas del desarrollo capitalista -especialmente el norte y el litoral mesopotámico-.El grueso de estos trabajadores se incorporaba a alguna de las múltiples empresas constructoras que llevaban adelante la instalación de la planta y de sus principales equipos. A su vez, y reclutados directamente por la empresa siderúrgica, arribaban a la ciudad profesionales, técnicos calificados y gerentes empleados por las firmas que brindaban la asesoría técnica y dirigían la instalación de la planta. ${ }^{10}$

La empresa siderúrgica tuvo políticas de reclutamiento y fijación de la fuerza de trabajo, totalmente diferenciadas hacia ambos contingentes de trabajadores que remiten a la configuración de un mercado de trabajo relativamente cerrado para técnicos y profesionales -mediante el reclutamiento selectivo y la garantía de la vivienda- y uno totalmente abierto en el caso de los obreros semi o no calificados -que arribaban a la ciudad sin garantías de empleo o vivienda.

Así, junto con la construcción de los primeros fundamentos de la planta industrial, se encara la construcción de un Barrio Residencial en las proximidades de la planta, para albergar a técnicos y profesionales extranjeros (empleados por las empresas que brindaban la asesoría técnica) y argentinos, que realizaban su proceso de formación y especialización en la industria siderúrgica. Se trata de un extenso barrio organizado alrededor de una avenida (Av. Central Malvinas Argentinas) que conduce a varios subconjuntos de viviendas, clasificadas por la empresa en cuatro subgrupos. Las calles transversales están numeradas correlativamente. El cruce de Avenida Central y la calle 21 es al mismo tiempo la división entre los sectores $1-2$ (compuestos por casas más grandes, ubicadas en terrenos amplios y parquizados y destinadas a gerentes y subgerentes) y los sectores 3 y 4 (destinados a las Jefaturas de División, de sectores, supervisores y obreros calificados). En este último y a la altura de la calle $\mathrm{N}^{\circ} 32$ sobre la Av. Central se ubicaban el núcleo "cívico - comercial"

Esplendor y ocaso de La Emilia Industrias Textiles SA Trabajo presentado en IV Encuentro Historia Regional del Sur Santafesino y el Norte Bonaerense. Theobald 1999. <http://www.alipso.com/monografias/fabrica_textil_de_la_emilia/> [Consulta: 7 de abril de 2010].

10 La población consignada en los Censos para San Nicolás de los Arroyos evidencia un crecimiento acelerado y coincidente con los procesos de expansión de la planta Siderúrgica. Para 1947, el partido contaba con 39000 habitantes, y el Censo de 1980 consigna 114000. En el mismo lapso de tiempo (1947 - 1980) la población potencialmente activa (es decir, en edad de trabajar independientemente de su situación laboral) pasa de menos de 30000 personas a aproximadamente 75000. SOUL, Julia, Relaciones de clase ...,Op. Cit, p. 69 ss. 
(estafeta postal, el local central de la Cooperativa de Consumo) y en la esquina de la calle 27, la delegación policial. Durante los '60 y los '70 se fueron construyendo las escuelas, la Iglesia y el club. La Memoria de 1958 consigna la cantidad de viviendas proyectadas: 24 de categoría superior. Las de categoría intermedia son 134 y las de categoría inferior son 484. Contrastando estos datos con los de dotación de personal -que comienza a ser consignada en el Ejercicio $\mathrm{N}^{\circ} 16-$ tenemos 642 viviendas de diversa categoría, para un total de 5015 trabajadores de todas las jerarquías. ${ }^{11}$

La concepción, planificación y la gestión del barrio hasta 1975 -momento en que la empresa pone a la venta las viviendas y comienza un lento proceso de municipalización- toman la forma de un company town restringido a un estrato particular de trabajadores siderúrgicos: aquellos técnicos y profesionales destinados a comandar la gestión del proceso productivo. La instalación del barrio redundaría en la inmovilización de la fuerza de trabajo a través de la vivienda ${ }^{12}$ con la expectativa de atraerla y fijarla al territorio en unas condiciones que resultaran atractivas y ventajosas, en un contexto de crecientes oportunidades de inserción laboral para profesionales y técnicos, dinamizadas por la profundización de la industrialización mediante la instalación de industrias manufactureras de capital extranjero, por lo que la competencia por la mano de obra calificada se incrementaba. ${ }^{13}$ Adicionalmente, la necesidad -particularmente importante en los inicios de las operaciones- de disponer constantemente de dichos trabajadores ante imprevistos y emergencias se veía satisfecha por este conjunto de viviendas cercano a la planta.

De esta manera, la instalación del barrio proyectó, mediante la incorporación al mismo de estratos bien definidos y jerarquizados, la segmentación propia de la organización del proceso productivo en el espacio urbano y produjo condiciones de vida y trabajo profundamente diferenciadas entre los trabajadores calificados y especializados y el resto de obreros de la empresa. La proyección social de las jerarquías fabriles operaba mediante la distinción de "los que viven en el barrio" y aquellos que no lo hacen. En este contexto, la función disciplinante de la forma company town se veía relativizada. Antes bien, este tipo de intervención de la

11 El cálculo en la dotación de personal no tiene en cuenta a los empleados de SOMISA en Casa Central - ubicada en Capital Federal -.En las Memorias el personal se encuentra clasificado en seis categorías. El Ejercicio $\mathrm{N}^{\circ} 16$ consigna: gerentes y subgerentes (8); profesionales (79); técnicos (958); capataces (263) y operarios (3707). El número de viviendas no incluye las plazas disponibles en las gamelas o dormitorios de solteros. Posteriormente, estas gamelas fueron remodeladas y se convirtieron en las viviendas de menor porte del barrio, destinadas a los niveles inferiores de mando y a técnicos especializados.

12 LEITE LOPES, José Sergio,"Fabrica e vila...", Op. cit.

13 PERALTA RAMOS, Mónica, La economía política argentina. Poder y Clases Sociales (19302006), Fondo de Cultura Económica, Buenos Aires, 2007. 
empresa remite a la necesidad de mano de obra especializada y es reconstruida como una de las evidencias del carácter social de la empresa, como opuesto a su carácter de lucro. ${ }^{14}$

Respecto del resto de los trabajadores, las intervenciones empresarias generaron condiciones de reproducción que contribuyeron a particularizar al colectivo obrero. Los indicadores de singularización de los somiseros que emergen con más fuerza en los relatos refieren, en primer término, a los niveles salariales ${ }^{15} \mathrm{y}$ a una serie de intervenciones empresarias que garantizaban condiciones relativamente mejores para la reproducción de este grupo obrero. ${ }^{16}$ Una explicación para estas condiciones remite al momento de configuración del colectivo obrero. En efecto, la forma de Estado propia del nacionalismo industrialista supuso un proceso de integración subordinada de la clase obrera, a través de la institucionalización de un conjunto de conquistas: salarios mínimos, regulación del uso y la gestión de la fuerza de trabajo mediante negociaciones colectivas, libertad de agremiación, etc. Es así que un conjunto importante de aspectos de las relaciones entre trabajadores y empresa se hallaban regulados en el ámbito estatal, de modo que se sustraían al control arbitrario de las gerencias. Además, la consolidación de las organizaciones sindicales de carácter nacional y centralizado, acelerada desde la década del ' 40 y los crecientes niveles de sindicalización entre los obreros industriales nutrieron la emergencia de un colectivo de trabajo tempranamente agremiado - ya sea en la Unión Obrera Metalúrgica (UOM) o en la Unión Obrera de la Construcción (UOCRA)-. Sus condiciones de trabajo se hallaban reguladas por Convenios Colectivos y se registran intensos procesos de militancia, organización y movilización reivindicativa desde la inauguración de la empresa. Consecuentemente, es posible identificar reivindicaciones sindicales detrás de cada intervención empresaria sobre la reproducción de los estratos obreros de la fuerza de trabajo. Esto es así con el establecimiento del refrigerio y de los comedores subsidiados, de la cooperativa de consumo y del sistema de transporte. Del mismo modo, se registran constantes reivindicaciones de aplicación de cláusulas puntuales del convenio colectivo vigente, así como demandas salariales y de condiciones de trabajo que tienen como referencia al conjunto del gremio metalúrgico. ${ }^{17}$

14 SOUL, Julia, Los unos y los otros. La fractura que persiste. Reconversión productiva e identidades colectivas en la ex SOMISA actual Siderar, Tesis de Licenciatura en Antropología, UNR, Rosario, 2002 (mimeo). SOUL, Julia, Relaciones de clase ...,Op. Cit.

15 Hemos mostrado cómo - aunque la política salarial de la empresa comprendía una serie de adicionales y premios- lo que contribuía notablemente a engrosar los salarios era la masiva realización de horas extraordinarias - en general de dobles jornadas. Ibídem.

16 Esta particularización también se fue sosteniendo y reproduciendo en el marco de la estrategia sindical dominante en SOMISA. SOUL, Julia, "La estructuración de una estrategia gremial dominante en SOMISA (1960 - 1976). Los procesos sindicales y las relaciones de hegemonía/subalternidad", en SIMONASSI Silvia y DICOSIMO Daniel (compiladores) Trabajadores y empresarios en la Argentina del siglo XX: indagaciones desde la historia social, Prohistoria, Rosario, 2011.

17 Además de entrevistas y relatos a militantes sindicales, estos procesos 'moleculares' pueden 
Socialmente, estas condiciones de reproducción se expresaron en la particularización de los somiseros como consumidores, evidente en los relatos que rememoran las facilidades para obtener créditos y para comprar a plazos en los comercios locales; apreciación corroborada por publicidades que interpelaban especialmente a los "empleados de SOMISA" para la venta de terrenos, automóviles o bienes durables. A su vez, esta importante masa salarial volcada al consumo local contribuyó al desarrollo de una importante estructura comercial y de servicios en la ciudad.

Subjetivamente, estas condiciones diferenciadas se expresan en términos contradictorios que, por una parte remiten al orgullo profesional -compartido con profesionales y jerárquicos- por participar de una actividad estratégica para el desarrollo nacional y a los saberes compartidos y a los procesos de movilidad social y bienestar asociados a la condición obrera. Pero por otra parte, las condiciones de trabajo y las marcas en el cuerpo (malformaciones físicas producto de la repetición de movimientos o posturas, mutilaciones producto de accidentes) y los padecimientos vinculados con la actividad laboral (enfermedades crónicas producto de la exposición al calor y por los turnos rotativos) son categorizados en clave de sacrificios por parte de los somiseros. Así, la identificación de los somiseros en tanto grupo obrero particular comprende un conjunto contradictorio de representaciones, que refieren a la persistencia de la explotación como supuesto de las mejores condiciones relativas de reproducción. Esta articulación se expresa mediante las construcciones sociosimbólicas propias de la integración subordinada de la clase obrera al proceso hegemónico del nacionalismo industrialista: nacionalismo, expectativas de salario justo y representaciones en torno de la dignidad del trabajador que se traducían en la legitimación técnica de las relaciones de jerarquía y autoridad fabriles, así como de sus límites, especialmente en relación con el uso de la fuerza de trabajo.

\section{Los somiseros en la ciudad. Intervenciones estatales y prácticas obreras}

La instalación de SOMISA sobreviene en un período de intensa transformación del Estado y de la sociedad argentinas, en plena consolidación de un proceso hegemónico que hemos caracterizado como nacionalismo industrialista. Uno de las características que asumió este proceso fue la centralidad del Estado Nacional en la dinamización de procesos de acumulación del capital industrial, específicamente en industrias básicas y estratégicas. Es en este marco que se produce la creación de SOMISA, como parte de un plan para alcanzar

inferirse de las Bases para la discusión de un convenio interno de SOMISA, en Archivo DIPBA (Dirección de Inteligencia de la Policía de la Provincia de Buenos Aires), Mesa B, Carpeta No 111, Leg $\mathrm{N}^{\circ}$ 43: fs $7-9$ y de las memorias empresarias de los Ejercicios de la década del ' 60 . Aunque no es el tema de este artículo, es necesario mencionar que registramos el predomino de políticas y nociones antisindicales entre los mandos medios y jerárquicos de la empresa. 
el autoabastecimiento siderúrgico. De modo que una primera forma de intervención estatal, está dada por la particular articulación con el capital privado (nacional y extranjero) para la creación de la empresa. Los planes estatales preveían mecanismos de reclutamiento, formación y entrenamiento de la fuerza de trabajo especializada y la instalación del barrio remite directamente a esa necesidad. ${ }^{18}$

Sin embargo, la masa de trabajadores que arribaban a la ciudad atraídos por la instalación de la empresa se instalaban en grandes extensiones de terrenos fiscales, formando villas miserias o adquirían lotes situados en antiguos terrenos de quintas y viñedos, conformando barrios obreros en el área que rodeaba al casco urbano. ${ }^{19}$ El rápido proceso de urbanización acrecentó el capital inmobiliario, en una dinámica que se tornó rápidamente especulativa, caracterizada por una rápida expansión de barrios obreros -mediante loteos y ventas informales- que se adelantaba en mucho a la expansión de los servicios y la infraestructura urbana.

El problema de la vivienda es una constante durante la década del ' 60 cuando la implementación de diversos planes habitacionales por parte del Estado no logró cubrir el déficit habitacional. ${ }^{20}$ Una etnografía situada en los barrios que se fueron configurando desde la década del ' 50 evidencia procesos de loteo acelerados y permite identificar una de las estrategias nodales que los obreros industriales pusieron en marcha para resolver la cuestión de la vivienda: las redes de ayuda mutua y los procesos de autoconstrucción. Los loteos acelerados y los procesos de autoconstrucción como mecanismos de edificación de la vivienda por parte de los obreros se yuxtaponen a las articulaciones en el ámbito institucional - estatal, que desde mediados de la década del ' 60 , se expresan en la implementación de diversos planes de vivienda por parte del Estado (provincial y municipal). ${ }^{21} \mathrm{El}$ conjunto de datos construidos a lo largo del trabajo de campo, permite establecer que las transformaciones socioespaciales en la ciudad de San Nicolás se produjeron predominantemente, mediante relaciones de mercado mediadas por la intervención estatal y recién en 1975 se registró la intervención de SOMISA

18 SAVIO, Manuel Nicolás, Obras Completas, Edición SOMISA, Buenos Aires, 1973.

19 Según el Plan Director "los propietarios de quintas o chacras suburbanas ... 'urbanizaron' pequeñas extensiones independientemente unas de otras y con fines especulativos individuales", PLAN DIRECTOR, Informe de Diagnóstico, Rosario. 1958 p. 297. La conformación de villas miseria en las cercanías del casco urbano fue motivo de conflictos, organización y movilizaciones ante planes de erradicación desarrollados por los gobiernos militares.

20 El registro periodístico del matutino local El Norte permite relevar numerosos anuncios y publicidades de loteos, planes de financiación para la compra de terrenos y construcciones de viviendas que dan cuenta de la intensa movilidad del mercado inmobiliario durante los sesenta.

21 Además de la Confederación General del Trabajo (en adelante, CGT), participan en la gestión de estos planes las organizaciones que agrupan a los empleados de comercio, a los trabajadores municipales, a los textiles y los metalúrgicos. Para una reseña completa de los planes de vivienda implementados en San Nicolás desde la década del ' 60 hasta los ' 80 ver SOUL, Julia, Relaciones de clase y construcción..., Op. Cit., p. 83 ss. 
en la cuestión de la vivienda obrera, cuando adquiere y cede a la organización sindical y al $\mathrm{FoNaVi}^{22}$ los terrenos para la construcción del "Barrio 7 de Septiembre".

El proceso de instalación de los trabajadores industriales provenientes de interior del país fue determinante en la producción de un entramado urbano segmentado en clave clasista. Los barrios situados en las cercanías del casco urbano histórico y el Barrio SOMISA -habitados por profesionales, propietarios rurales e industriales o comerciantes- con plena provisión de los servicios urbanos, buenas vías de comunicación y sistemas de transporte adecuados se diferenciaban de aquellos en que se había establecido el grueso de los trabajadores, no sólo por su ubicación geográfica

- distantes del casco urbano histórico- sino también porque éstos últimos aún en la actualidad carecen de servicios e infraestructura urbana (como pavimento, alumbrado público, servicio de cloacas o agua potable), el sistema de transporte y comunicación con el centro de la ciudad resulta deficiente y el establecimiento de instituciones sanitarias y educativas es tardío. Esa situación fue la base material para el desarrollo de instituciones y organizaciones tendientes a resolver los problemas de infraestructura urbana.

En una mirada que atienda al proceso hegemónico, estas instituciones entraman las prácticas que configuran los subalternos en pos de satisfacer sus necesidades inmediatas en el sistema institucional-estatal y, de esta manera constituyen y expresan las relaciones de hegemonía- subalternidad. Así, este entramado de prácticas e instituciones fue el medio a través del cual se estructuraron procesos de participación social, por medio de los cuales los trabajadores construyeron, plantearon y disputaron la consecución de sus demandas con el Estado en sus múltiples expresiones. En este sentido, es relevante el rol de las Asociaciones Vecinales, cuya creación fue el modo de vehiculizar demandas urbanas frente al Estado Municipal. Las Asociaciones Vecinales se convirtieron en los actores protagónicos de la gestión de obras vinculadas con la provisión de servicios públicos, asfalto, la mejora de calles, la realización de cordones cuneta, colocación de teléfonos públicos, gestión de salas de primeros auxilios o dispensarios, regularización urbana y dominial, etc. Al mismo tiempo eran las entidades encargadas de la organización de eventos sociales en el barrio: despedidas de año, fiestas de carnaval, cenas navideñas.

Estas prácticas que los trabajadores desarrollan en tanto ciudadanos o vecinos, tienen una relación exterior con la empresa, sin que registremos políticas o intervenciones específicas respecto de las demandas de los vecinalistas. Sin embargo, muchos de los somiseros y sus familias fueron activos participantes en las asociaciones vecinales de sus barrios y solían recurrir a la empresa en pos de gestiones puntuales - como donaciones o ventas de materiales a bajo costo- primero informalmente y luego por los canales institucionales correspondientes. ${ }^{23}$

22 Fondo Nacional de la Vivienda.

23 En un núcleo problemático que no podemos explorar aquí, mencionamos que numerosos vecinalistas otorgan relevancia a las articulaciones estatales durante el período de la Dictadura Mili- 
En el caso de los somiseros, a los procesos de participación en las Asociaciones Vecinales se suma el entramado institucional en el que se inscriben a partir de su sindicalización, en un contexto de orientación de la estrategia de la UOM - y de las organizaciones sindicales en general- hacia la gestión de servicios sociales y de intervención en la esfera de la reproducción de la fuerza de trabajo. Además de los planes de vivienda, la organización sindical incrementó los servicios de salud, inauguró centros educativos con orientación técnica y otros destinados a niños con capacidades diferentes y contribuyó activamente en la gestión de las políticas sociales de la empresa. ${ }^{24}$

De manera que las políticas empresarias contribuyeron a crear un colectivo obrero particularizado respecto del conjunto de trabajadores industriales de la región. Esta particularización no opera a través de relaciones obrero- patronales de características de la gran industria -anonimato, asociaciones corporativas, regulación estatal-y remite a las formas e instituciones vinculadas con la reproducción de la fuerza de trabajo y con la construcción de los somiseros como consumidores.

Desde la perspectiva de los actores locales, los profundos procesos de transformación que supuso la instalación de SOMISA y la afluencia de trabajadores a la ciudad y el crecimiento acelerado y desorganizado, fueron procesados a nivel local en clave de la contradicción, poder central/poder local, y su equivalente, factores exógenos/factores locales. En su dimensión ideológica y cultural, estas tensiones se expresaron en la dualidad nicoleños y somiseros como clivaje identitario.

\section{Nicoleños y somiseros o de lo exógeno y lo local como clivajes sociales}

La activa presencia estatal en los intentos de direccionalidad del proceso socioeconómico, implicaba la preocupación por procesar y resolver las tensiones locales generadas por el proceso de industrialización. En este sentido, el Estado en su nivel local -tanto Municipal como sus representantes en el Poder Legislativo Provincial-dinamiza iniciativas vinculadas con la producción de San Nicolás como epicentro regional. Una de ellas, en consonancia con otras políticas similares de la época, es la elaboración de un Plan Director que ya en

tar de 1976-1983, ya que -de la mano de la Ley de Ordenamiento Territorial de 1977 encontraron eco muchas de las demandas históricas de regularización urbana por parte de estas asociaciones. El Arquitecto Eduardo Bluhn aporta algunos elementos que explican esta importancia, al sostener que la Ley de Ordenamiento Territorial y Uso del Suelo No 8912, dictada por el Gobierno Militar de la Provincia de Buenos Aires frenó el crecimiento de San Nicolás en extensión, al reclasificar las tierras y detener -aunque parcialmente- la especulación. BLUHN, Eduardo, La intervención del Estado en los procesos de Desarrollo Urbano como factor de integración física y social. El caso de la ciudad de San Nicolás en el período 1950-2000, Tesina final de la Carrera de Especialización en gestión local del hábitat popular (mimeo), San Nicolás, 2006.

24 El Norte, Suplemento Especial, San Nicolás, 25/05/1983. 
1958, preveía la regulación y planificación del proceso que se estaba desarrollando. De este documento -inscripto en las coordenadas políticas e ideológicas del desarrollismo-se desprende la apuesta de los diferentes niveles del Estado por la complementariedad de su accionar y por la planificación como vectores en la dirección del proceso socioeconómico. De esta manera, el Plan postula un conjunto de acciones de coordinación cuyo principal actor es el Estado en su nivel municipal. ${ }^{25}$ La presentación del plan realiza un diagnóstico preciso de la situación, al indicar que la ciudad "vive un momento crítico de su evolución. La tranquila ciudad colonial de hace una generación aprecia los beneficios de la industrialización, pero comienza a sentir los peligros de un incontrolado uso de la tierra y la falta de precisas directivas" ${ }^{26}$ El objetivo del Plan es que la ciudad se convierta en el centro regional de circulación de mercancías dinamizado por el Puerto, dado que el desarrollo industrial estaba garantizado por el proceso desencadenado por la instalación de la siderúrgica. Entretanto, los legisladores provinciales realizaban intervenciones tendientes a lograr la optimización de posibilidades de inversión en la localidad. La principal en esta dirección fue el Proyecto, presentado por el Diputado Rodríguez Araya de declarar zona industrial al Puerto y a terrenos aledaños, con el fin de facilitar allí la radicación de industrias. ${ }^{27}$ Aunque ambas iniciativas corrieron suertes diversas, fueron absorbidas por otras políticas y redundaron en la instalación de empresas pequeñas y medianas, no lograron contrarrestar el carácter dominante de SOMISA en la configuración de la dinámica socioeconómica de la región.

En este sentido, las Editoriales publicadas por el periódico local El Norte durante la década del '60 evidencian la construcción discursiva de la ciudad como un actor con intereses particulares y diferenciados de los que expresa el accionar de la empresa, fruto de decisiones centralizadas de las que no participan los actores locales. Estos reclamos se formulaban en nombre del progreso de San Nicolás, reivindicando así el rol fundante de la ciudad -más precisamente de sus clases y elites dominantes- en la construcción del Estado y de la nación. En las historias locales de la Ciudad el rol de las elites locales -particularmente del Comisionado Municipal, Román Subizaes- fundamental para la decisión estatal de instalar la planta en San Nicolás. ${ }^{28}$ Así, el repetido reclamo del editorialista por la participación nicoleña en la toma de decisiones, recupera esta agencia como causa de la instalación de la planta como argumento

25 Algunos elementos teóricos que sustentan estas iniciativas se hallan sintetizados en ROFMAN Alejandro y ROMERO, Luis, Sistema socioeconómico y estructura regional en Argentina, Editorial Amorrortu, Buenos Aires, 1973.

26 PLAN DIRECTOR..., Op. Cit, p. 2.

27 El Norte, San Nicolás, 20/04/ 1961.

28 Una breve revisión de las narrativas de las clases dominantes locales en SOUL, Julia, Relaciones de clase ..., Op. Cit., p. 61 ss. 
... en la práctica, en el espíritu y en las necesidades San Nicolás ... no ha intervenido ni interviene para nada ... en su estudio [de los planes de expansión de SOMISA]... deberían estar obligados a intervenir porque San Nicolás fue y es la razón histórica, geográfica y moral de la presencia de esta gran planta ... Ramallo que ... reclama la ubicación, la ventaja y los bienes de todas las posibilidades ... [San Nicolás] reclama justicieramente ser cabeza en el estudio de los grandes planes y no invitada de piedra. ${ }^{29}$

Localmente se fue configurando un campo de fuerzas sociales aglutinadas en torno del clivaje exógeno / local, que caracterizaría con su impronta las relaciones sociales de la ciudad. Esta dicotomía se convirtió en una clasificación que distinguía a los obreros siderúrgicos del resto de la población mediante las identificaciones de somiseros y nicoleños. Los primeros, trabajadores industriales, llegados desde las provincias, encontraron en la empresa un empleo estable y en San Nicolás una ciudad donde instalarse con sus familias, construir barrios e integrarse a asociaciones vecinales, clubes, organizaciones sindicales y políticas. Los segundos vieron cómo lo promisorio del desarrollo industrial significaba a su vez, crecimiento urbano desordenado, especulación inmobiliaria y precios altos en las mercancías destinadas al consumo doméstico, al mismo tiempo que esa masa salarial volcada a la circulación era un elemento central en el progreso de la ciudad.

La experiencia de los nicoleños, narrada en términos del pasaje de una ciudad apacible y armónica a una ciudad industrial es reconstruida con una sensibilidad nostálgica, en la que predomina el recuerdo de la instalación de SOMISA como una disrupción de este pasado idealizado. Además los relatos expresan otro conjunto de transformaciones sociales asociadas con la industrialización y son aquellas que se vinculan con la puesta en cuestión de los indicadores de estatus en el seno de una comunidad en la que el prestigio social se asociaba fuertemente a la propiedad inmobiliaria y a las profesiones liberales. ${ }^{30}$ Un relato del proceso de radicación de los somiseros por parte de una nicoleña da cuenta de estas valorizaciones:

Llegaban en los trenes... ahí no más los cargaban y los llevaban en camiones hasta la planta... Y entraban! En seguida... ya tenían trabajo. Y cuando salían de la fábrica... imagínate todo el playón con las mesitas y las sombrillas... los que loteaban... y le decían "vino para trabajar acá?, y se va a quedar? Y tiene casa?!” y ahí no más le vendían... (...) Y bueno, eso resultó que gente que a lo mejor no tenía ni segundo grado, después tenía dos o tres propiedades, porque iban comprando y a lo mejor vivían en una piecita y alquilaban o vivían en una y pagaban la cuota de otro terreno... Entonces te encontrás con eso... gente que no tiene ni la [educación] primaria [completa] y a lo mejor vive de rentas.

La contrariedad expresada por la funcionaria entrevistada por la paradoja de ser un obrero

29 El Norte, San Nicolás 05/11/1964.

30 ARENSBERG, Conrad, "Industry and Community" en American Journal of Sociology, 1942, $\mathrm{N}^{\circ} 48$. 
industrial y vivir de rentas indica la subversión en los indicadores del status que supuso la instalación de la empresa en la ciudad. Si bien no es directamente el tema de este artículo, es relevante mencionar que esta contrariedad permanecerá como contenido de apelativos como los negros o la negrada, que resultan dominantes hacia los somiseros. ${ }^{31}$ Lo que resulta relevante para nuestra indagación es que las prácticas obreras, al mismo tiempo que disruptivas de estatus, son categorizadas como una de las principales causas de la problemática urbana nicoleña y, consecuentemente, objeto de intervención, regulación y normativización. En este sentido, los trabajadores migrantes -centralmente futuros somiseros- son construidos como otros -extraños de los nicoleños tanto en lo relativo a su inserción económico- productiva como en referencia a sus prácticas políticas y culturales, otros que ocupan una parte definida del espacio urbano: aquella que no se encuentra plenamente integrada a la ciudad. Las Asociaciones Vecinales son las herramientas del ámbito institucional - estatal mediante las cuales los somiseros -y los obreros industriales en particular- procuraron viabilizar su integración a la ciudad y la mejora de sus condiciones de vida.

Las características que distinguían a los somiseros y que -por extensión- otorgaron a San Nicolás su fisonomía de ciudad industrial, resultaban de articulaciones concretas de las prácticas de los trabajadores en las instituciones (sindicatos, asociaciones vecinales, etc.) y de la forma de Estado propia del nacionalismo industrialista. Estas articulaciones y entramados entraron en crisis sostenidamente desde la década del ' $70 \mathrm{y}$ fueron desplazadas definitivamente con la consolidación del proceso hegemónico neoliberal, que en San Nicolás tuvo como hito la privatización de SOMISA.

31 Los procesos de estigmatización vinculan causalmente, atributos sociológicos de los trabajadores industriales -su carácter de migrantes rurales, su falta de educación formal y de experiencia industrial y urbana - con prácticas productivas, gremiales y sociales mediante nociones que relativizan el carácter 'moderno' y 'racional' de sus acciones. Estos procesos se sintetizan en diferentes apelativos peyorativos, que tienen como referencia a grupos sociales que conforman los contingentes de trabajadores no calificados y semi - calificados, base de la organización industrial. SOUL Julia y VOGELMANN Verónica, "Interrogando al sentido común desde las relaciones de hegemonía. Aproximación antropológica a los procesos de estigmatización de trabajadores industriales", en Dimensión Antropológica ,Instituto Nacional de Antropología e Historia, México (en prensa), № 57. También es menester mencionar que dichos procesos son la expresión particular de los procesos de estigmatización de trabajadores industriales constitutivos del sentido común en las sociedades capitalistas .MENENDEZ Eduardo, Antropología Médica. Orientaciones, desigualdades y transacciones, Cuadernos de la Casa Chata, México, 1990.

\section{6}




\section{El estallido de los somiseros en el proceso hegemónico neoliberal: crisis y reconversión productiva}

La privatización de SOMISA fue el hito que marcó la culminación local de los procesos de reestructuración capitalista y Reforma del Estado que se desplegaban en el país desde mediados de la década del '70. Las consecuencias inmediatas más importantes para los somiseros, fueron la pérdida de más de 6000 puestos de trabajo y la irrupción del patrón en la planta ${ }^{32}$ en virtud de la adquisición de la empresa por parte del grupo empresario Techint. Durante 1995, la planta pasa a ser parte de Siderar - una red de plantas localizadas en diferentes puntos del país-y posteriormente, de Ternium - consorcio siderúrgico emplazado en varios países de América Latina. De modo que mediante la privatización se realizaba un proceso de concentración y centralización de capitales siderúrgicos, que culminarían en la configuración de una empresa transnacional, cuyas principales decisiones se toman en función del mercado internacional.

La coyuntura de la crisis reveló los alcances de la paulatina imposición de un nuevo sentido común que cuestionaba a lo estatal y responsabilizaba a los trabajadores del Estado por la debacle del mismo, la dupla nicoleños/somiseros se reconfiguró. La crisis evidenció los vínculos necesarios entre lo exógeno y lo local. En este sentido, el amplio consenso crítico hacia las empresas estatales y sus trabajadores se contraponía con la evidencia de que los somiseros, como grupo obrero, dinamizaban la vida económica de la ciudad y que el desempleo y la racionalización acarrearían consecuencias sociales que afectarían al conjunto de la 'comunidad'. De esta forma, la reconversión productiva con paz social articulaba las reivindicaciones e intereses de nicoleños y somiseros hacia el Estado nacional, articulación expresada organizativamente por el Consejo Regional para la Defensa de SOMISA y del Patrimonio Nacional. En este sentido, las construcciones simbólicas que articularon el consenso social frente a la privatización son la expresión local del proceso de crisis que sienta las bases para la ofensiva del capital, expresadas mediante las construcciones socioideológicas que establecían la imposibilidad de las empresas estatales de ser eficientes y otorgaban importancia central y revitalizadora del capital privado. ${ }^{33}$

32 Hemos analizado estas transformaciones en la perspectiva de los trabajadores siderúrgicos en SOUL, Julia, "Procesos hegemónicos y cotidianeidad. Prácticas obreras en la privatización de la Sociedad Mixta Siderúrgica Argentina", en Cuadernos de Antropología Social, Sección de Antropología Social. FFyL UBA, Julio 2009, № 29. y SOUL, Julia, Los unos y los otros..., Op. Cit.

33 BONNET, Alberto, La hegemonía menemista. El neoconservadurismo en Argentina (1989-2001), Editorial Prometeo, Buenos Aires, 2007. PIVA, Adrián, Acumulación y hegemonía en la Argentina menemista, Editorial Biblos, Buenos Aires, 2012. Para las expresiones particulares en los territorios de emplazamiento de grandes empresas estatales: RIVERO Cynthia y GOURNALOUSSE Juan, "Confusión organizada, arbitrariedad consciente. Imágenes en torno a la deslegitimación de las empresas del Estado", Ponencia presentada en $8^{\circ}$ Jornadas Rosarinas de Antropología Sociocultural, Rosario. 
La privatización de la empresa inaugura un período de ofensiva del capital respecto del trabajo -que se desarrolló con mayor intensidad durante la primera mitad de la década del ' 90 - cuyos elementos centrales continúan desplegándose en la actualidad. La toma de posesión por parte de los nuevos propietarios supuso la reinscripción del colectivo somisero en un proceso hegemónico que tenía como pilar la transformación de las formas de uso y gestión de la fuerza de trabajo y -por ende- de las coordenadas políticas y socio-ideológicas desde las cuales se producía la integración de los subalternos al mismo. En los relatos de los cuadros gerenciales, esta importancia adquiere el tono de refundación de las relaciones sobre nuevas bases.

La particularización de los somiseros en el ámbito de la reproducción continúa operando en el proceso hegemónico neoliberal, reconfigurada en diferentes articulaciones y en una correlación de fuerzas netamente regresivas para la clase trabajadora. Esta particularización forma parte de fuertes procesos de segmentación en términos salariales, contractuales y de condiciones de trabajo entre diversos conjuntos obreros: los empleados por empresas tercerizadas y subcontratistas, los nuevos trabajadores de planta y los antiguos somiseros que continúan siendo identificados como un grupo singular al interior de la fábrica, en virtud de ciertas condiciones de trabajo y prácticas productivas, además de su participación en el Programa de Propiedad Participada (PPP). ${ }^{34}$

Sin embargo, los nuevos propietarios pusieron en marcha políticas de recorte y ajuste de un conjunto de intervenciones que habían garantizado aquellas condiciones particulares de reproducción del colectivo somisero, categorizadas como beneficios y como obstáculos para la competitividad de la empresa. La noción de beneficios presenta importantes continuidades con contenidos propios del sentido común que sustentó el consenso social hacia el proceso racionalizador. En el contexto de crisis previo a la privatización de la empresa, las condiciones particulares de reproducción que distinguían a los somiseros eran categorizadas como beneficios o privilegios que conspiraban contra el desarrollo de la empresa. Esta noción como legitimadora de políticas empresarias regresivas expresaba la dimensión ideológica cultural del consenso social a la privatización. La imposibilidad de percibir esos beneficios se asocia, en el discurso y en las políticas empresarias, con la noción de que los trabajadores no se los ganaron; no hicieron ningún esfuerzo para recibirlos $\mathrm{y}$, por lo tanto, no los valoran. De modo que las políticas de avance sobre conquistas sindicales

2007. El desarrollo de este proceso al interior de la empresa en SOUL, Julia, "Procesos hegemónicos ...” Op. Cit.; SOUL, Julia, Los unos y los otros..., Op. Cit.

34 La Ley 23.696 de Reforma del Estado estableció un Programa de Propiedad Participada para las empresas destinadas a ser privatizadas. Para ello establecía en su artículo 29 que el ente a privatizar debía emitir bonos de participación en las ganancias para el personal en función de su remuneración, su antigüedad y sus cargas de familia. Esta integración de los antiguos somiseros en clave de propietarios es un potente recurso de desmovilización gremial, aunque no promueve particulares formas de adhesión subjetiva a la empresa.

\section{8}


tanto en el terreno de la producción como en el de la reproducción, se realizan en nombre del crecimiento con desarrollo tal como lo relata uno de los directivos de la empresa, al correlacionar causalmente la implementación de la polivalencia en el proceso productivo con la generación de beneficios.

Nosotros teníamos que ir dando los beneficios de a poco. Fuimos creando esos beneficios. El primer mensaje era que habia que laburar de manera tal que los beneficios los generáramos. Bueno, dicho y hecho, tal es así que para el primer año la empresa no perdió esos 30 millones que habíamos pensado sino que salió hecha. Para el segundo año se ganaron 30 millones y para el tercer y cuarto año la suma ascendió [...] La idea de esta empresa es que si vos sos de producción te tenés que hacer cargo del control de calidad y de mantenimiento. Así se aglutinan tareas y se facilitan las cosas. A partir de este punto, al cambiarse todo, se define el nuevo Plan de Beneficios y Desarrollo Social. El beneficio no tiene que ser la contraprestación económica si ésta no aporta al desarrollo. Mi objetivo era que el agente fuera percibiendo que iba a tener beneficios en función de cuánto rindiera la empresa. " 35

Las intervenciones de los nuevos propietarios respecto de los somiseros adquieren un sentido opuesto a las que habían caracterizado al período de gestión estatal, explicitando y legitimando el lucro, el negocio y la rentabilidad como principios rectores de las relaciones fabriles. Este conjunto de significaciones será el sustento de intervenciones empresarias tanto hacia el conjunto de trabajadores como a la comunidad.

Respecto del colectivo obrero, con la privatización de la empresa, se produjo la municipalización definitiva del barrio, con las consecuentes transformaciones en el antiguo enclave somisero: la llegada de nuevos propietarios nicoleños que adquirían las propiedades, la inclusión del barrio en el esquema de los servicios urbanos municipales que significaba cargas impositivas para sus habitantes (recolección de residuos, alumbrado público, etc.). A su vez, los nuevos propietarios de la empresa reservaron el terreno que separaba los barrios $1-2$ y $3-4$, para emplazar allí un conjunto de viviendas construidas con estructuras de acero. Este modo intervención de la empresa en la cuestión de la vivienda no pretende recuperar formas propias del company town, sino que se produce directamente bajo la forma mercantil: las viviendas se construyen como mercancías, y las operaciones de venta permanecen en la órbita de la empresa privatizada. Los destinatarios (empleados y funcionarios de la misma) perciben condiciones especiales crediticias y de financiamiento bajo la lógica de ayudar a quien se ayuda.

El mismo sentido informa los criterios que estructuran las intervenciones empresarias hacia los grupos familiares de los trabajadores: programas de becas en función de promedios y rendimiento académico, colonias de vacaciones y eventos para niños en función de cupos y desempeño, etc. Estas intervenciones empresarias, investidas de una forma pedagógica,

35 Ex Gerente de Relaciones con la comunidad. Siderar, 2012. Nuestro resaltado. 
son portadoras de un mensaje que torna incuestionables las nuevas relaciones laborales. El mensaje de que hay que generar los beneficios fijó, en un colectivo obrero diezmado por el desempleo, la incertidumbre y las consecuencias sociales de la reestructuración productiva, ${ }^{36}$ la correlación entre ganar y generar los beneficios como nociones equivalentes: el modo de ganar beneficios es generándolos, es decir, optimizando la rentabilidad empresaria. Se borran las huellas del accionar sindical como medio para ganar conquistas o derechos, privativo de los trabajadores y se consagra el principio de la rentabilidad y la reproducción del capital como vector de las formas de intervención del conjunto de los actores sociales. Esta operación político - ideológica es el sustento de las intervenciones empresarias en el ámbito de la comunidad.

\section{La Ciudad del Acero en el proceso hegemónico neoliberal: de emprendedores y SOMISA dependientes}

Si las décadas del ' 60 al ' 80 , enfrentaron a San Nicolás a problemáticas de infraestructura urbana y vivienda, la década del ' 90 tuvo como centro las problemáticas ocasionadas por el impacto de los procesos de reestructuración capitalista. En la ciudad de San Nicolás, las consecuencias del proceso de privatización y reconversión productiva se expresaban en una caída de U\$S 500000 del PBI regional para 1995, la generalización de situaciones de subocupación o desocupación a la mitad de la Población Económicamente Activa y el deterioro -y en algunos casos el colapso- de las intervenciones estatales en atención sanitaria e infraestructura básica. ${ }^{37}$

La crisis social producto de la reestructuración productiva fue canalizada tempranamente mediante un conjunto de intervenciones de organismos estatales y supraestatales ${ }^{38}$ que

36 RIVERO Cynthia y PALERMO Hernán, "Memorias del trabajo ante los procesos de privatización en Argentina", en Revista Nómadas, IESCO-UC, Bogotá, abril 2011, № 34; SOUL, Julia, “Acá lo que cambió todo fue la privatización. Aproximación antropológica a las prácticas obreras en los espacios laborales en procesos de privatización y reconversión productiva", en Revista Theomai , UNQ, $1^{\circ}$ semestre 2010, No 21 .

37 HERRERA Luis, Proyecto Reparación Patrimonial Histórica para San Nicolás. Antecedentes, julio 2003. p .2. Es en contextos similares - comunidades que habían crecido en torno a empresas estatales privatizadas que había disminuido abruptamente su dotación de personal tanto directo como indirecto, crisis en las economías regionales- que estallaron hacia 1996 las primeras movilizaciones de trabajadores desocupados, que irían focalizando sus reivindicaciones hacia la gestión de recursos sociales para la atención de necesidades alimentarias, sanitarias, de infraestructura urbana y para el desarrollo de emprendimientos productivos.

38 Entre la principales intervenciones relevadas - que condensan acciones de organismos públicos, privados y no gubernamentales- se pueden mencionar tres que revistieron particular importancia en la formulación de políticas regionales: Plan Estratégico de San Nicolás (PLESAN), Documento Base 
produjeron diagnósticos, evaluaciones y proyecciones alrededor de los procesos de reconversión de la fuerza de trabajo. En un sentido orgánico respecto del proceso hegemónico en curso, estas intervenciones daban lugar a políticas públicas focalizadas cuyo objeto sería la población de trabajadores desocupados. De modo que las calificaciones y actitudes de los trabajadores se tornaron objeto de minuciosos estudios, de los que surgió la contraposición emprendedores / SOMISA - dependientes. ${ }^{39}$ La importancia de esta contraposición es que sintetiza las transformaciones que se desarrollaron en la comunidad desde la década del ' 90 y que se proyecta tanto hacia atributos de individuos como hacia las capacidades de la comunidad. Los emprendedores son capaces de probar cosas nuevas, enfrentar entornos cambiantes y asumir desafíos en pos de lograr el progreso y el crecimiento, como una versión minimizada del entrepreneur schumpeteriano. Hacia ellos se dirigirán una serie de programas y políticas públicas destinados a reconvertir sus atributos laborales, a incentivar sus emprendimientos y negocios, viabilizados por dependencias estatales locales creadas para tales fines (como la Dirección de Empleo o la Agencia de Desarrollo Económico). En el otro extremo, los SOMISA dependientes no saben ver o aprovechar oportunidades, están pegados al pasado y no logran adaptarse a los cambios que imponen las nuevas épocas. La contraposición adquiere funcionalidad también para ubicar, en los atributos y actitudes de los SOMISA dependientes las causas de sus dificultades para reingresar al mercado de trabajo. Esta dicotomización de los trabajadores se expresaba, a su vez, en la configuración de intervenciones diferenciadas para los diferentes grupos.

Una mirada sobre la dinámica social durante la década del ' 90 evidencia la configuración de un entramado singular de instituciones de la sociedad civil (las organizaciones sindicales y las Asociaciones Vecinales) que vehiculizaron las prácticas de participación social de trabajadores y trabajadores desocupados. Las organizaciones sindicales desarrollaron un accionar de importancia respecto de los trabajadores desocupados, y este es un rasgo distintivo respecto de lo acontecido en otras regiones que sufrían las consecuencias de procesos de privatización. Este accionar consistió, centralmente, en asumir la gestión de los recursos provenientes de los programas de política pública focalizados, específicamente el Plan Trabajar y el Programa de Asistencia Solidaria (PROAS) del Ministerio de Trabajo y del Instituto Provincial de la Vivienda. Según las fuentes sindicales, de estos planes participaron casi mil trabajadores y trabajadoras que en general, se integraban a los proyectos a través de las organizaciones vecinales, en los barrios respectivos.

San Nicolás, 1999; Plan Estratégico de San Nicolás: Diagnóstico y Formulación 2002. Disponibles en http://www.ipusannicolas.gov.ar/docplesan.html [Consulta: 8 de mayo de 2010]; INSTITUTO INTERNACIONAL DE MEDIOAMBIENTE Y DESARROLLO Diagnóstico socioambiental de San Nicolás, PNUD, 1993.

39 En términos de proceso hegemónico, esta dicotomía resulta en un movimiento orgánico con lo que acontecía puertas adentro de la empresa, que premiaba al personal proactivo y que demostraba compromiso y capacidad de resolver los problemas que se le presentaban a la empresa 
Las Asociaciones Vecinales resultaron centrales en la coordinación de estas políticas. De una parte coordinaban con la CGT la organización de los emprendimientos productivos y se posicionaban frente al Estado Municipal como referentes para la gestión de otros recursos (bolsones de alimentos, colchones, chapas o materiales de construcción). Indagando sobre estas prácticas en dos Asociaciones Vecinales, registramos actividades relacionadas con la organización de emprendimientos de reciclado de ropa y comedores comunitarios. Además, asumían la función de identificar y convocar a los beneficiarios de Planes Sociales y de Capacitación Laboral.

Este entramado institucional que articula organizaciones vecinales y sindicales con programas estatales y supraestatales, dirige a todo un contingente de mano de obra que no lograría reconstruir una relación asalariada, hacia formas de trabajo productivo tendientes al intercambio a baja escala (huertas) o a la consecución de obras de infraestructura social que de otro modo debiera haber afrontado el Estado. De esta manera, la organización sindical redirecciona su accionar hacia los trabajadores desocupados, adaptando demandas y reivindicaciones a las características que asumen las políticas sociales en la forma de Estado neoliberal. Esta política permitió reproducir condiciones para procesar una crisis socioeconómica fabulosa, producto de la reestructuración productiva sin grandes episodios de conflictividad social. ${ }^{40}$

En este punto, la cuestión del desarrollo local sintetiza las relaciones que se generan entre Estado y sociedad civil en virtud de la aplicación de políticas focalizadas por parte de entidades estatales y no estatales de anclaje local. Dos son los principales organismos creados en la órbita del Estado Municipal, conducentes a la focalización y especialización de las políticas de desarrollo local. En primer término, relativo a la estructura socioproductiva, se dinamiza el Ente de Promoción del Plan COMIRSA (ente provincial del que participan los Intendentes de San Nicolás y Ramallo). ${ }^{41}$ Este ente gestiona y administra un parque industrial de 430 has. de superficie ubicado en frente de los terrenos de Siderar zonificado según el tamaño de las empresas. Una parte importante de la superficie del predio COMIRSA está ocupada por empresas de instalación relativamente reciente en la zona, surgidas al calor de

40 Este entramado institucional desarrolló acciones en tres áreas: servicios comunitarios, infraestructura y vivienda. Los Servicios comunitarios comprendieron, entre otros rubros, huertas comunitarias (con un programa específico), reciclado y confección de ropa, servicios sanitarios en prevención y control, preparación de comidas en comedores comunitarios. La Infraestructura comunitaria comprendió tareas de construcción de centros de salud, salones de usos múltiples, bibliotecas populares, iluminación y redes de alumbrado público, zanjas y alcantarillado. CGT SAN NICOLAS, Informe de Actividades: programas sociales organizados por la CGT San Nicolás, Marzo 2001 (mimeo).

41 Creado por ley No 11473 en 1993. Se trata de una entidad provincial que tiene como propósito fomentar la instalación de establecimientos industriales y de servicios directos a industrias. La instalación de una empresa allí implica exenciones impositivas, cesión de terrenos, provisión de infraestructura y servicios y otras facilidades establecidas en la Ley Provincial de Promoción Industrial № 10547. 
los procesos de tercerización que dinamizaron los nuevos propietarios de la planta siderúrgica. Su creación en 1993, es expresiva de la continuidad de políticas públicas destinadas a generar, atraer y optimizar las inversiones del capital industrial en la región.

En segundo lugar, el IPU (Instituto de Planeamiento Urbano), dependiente del Poder Ejecutivo Municipal encargado de la generación y el desarrollo de propuestas vinculadas con el Planeamiento Urbano y su coordinación con directivas de niveles nacionales o provinciales. El instrumento generado por el IPU para la producción de políticas urbanas es el Plan Estratégico San Nicolás (PLESAN). El PLESAN parte del diagnóstico y las necesidades de la ciudad de San Nicolás, a partir de la generación de consensos y concertación entre el ámbito público y el privado, con el objetivo de "diseñar y construir el desarrollo económico desde sus propias potencialidades y peculiaridades como territorio y como región" ${ }^{42}$ En contraste con el antiguo Plan Director, que suponía generar acciones de planificación centralizada y direccionamiento del proceso económico, el PLESAN tiene como norte la construcción de las potencialidades locales como incentivos para la inversión. Resultan relevantes las referencias del PLESAN a los actores de la sociedad civil-entre los cuales se cuentan asociaciones corporativas, la empresa privatizada y una multiplicidad de organizaciones e instituciones estatales y no gubernamentales expresivas de intereses particularescomo actores en relaciones de poder simétricas y la invisibilización de la centralidad que la empresa siderúrgica continúa teniendo en la vida económica y productiva de la región.

La empresa privatizada, a través de intervenciones generadas desde la Gerencia de Relaciones con la Comunidad, tiene un accionar autónomo y una política de articulación con entidades locales desarrollada en todos los territorios en que se emplazan sus plantas. Al tratarse de una empresa emplazada tanto en Argentina como en el exterior, el accionar 'hacia las comunidades' reviste un nivel mayor de centralización que el de SOMISA, y funciona como punta de lanza para la instalación en las comunidades de fundaciones y organizaciones no gubernamentales con las que coordina programas de acción. Las acciones más relevantes se registran en el área de Deportes -organización de maratones anuales y de campeonatos-Salud -charlas, talleres de formación, campañas-Educación-con activas articulaciones con escuelas técnicas y primarias-y Cultura -organización de ciclos de cine, aportes a la gestión estatal-. Mediante estas acciones, la empresa busca posicionarse como un actor relevante en la órbita de la comunidad. ${ }^{43}$ Estas líneas de intervención constituyen una ruptura importante respecto de la forma de gestión de la estatal SOMISA que centraba sus prácticas exclusivamente en los somiseros. Las intervenciones empresarias referidas a

42 PLESAN, Diagnóstico y Formulación, Instituto de Planeamiento Urbano de San Nicolás, 2002, p 22. Tanto el PLESAN como el IPU son expresiones a nivel local de la forma de Estado propia del proceso hegemónico neoliberal que, como tales, se replican en organismos similares en otras ciudades.

43 Algunas intervenciones de fuerte contenido simbólico, como el auspicio de la restauración del Teatro Rafael de Aguiar, tienden a construir referencia de modo directo. 
la comunidad remiten al despliegue de las estrategias de Responsabilidad Social Empresaria, dispositivo global que expresa las pretensiones hegemónicas de las empresas como actores sociales. ${ }^{44}$

De manera que, desde la década del ' 90 hasta la actualidad, el entramado institucional y político mediante el cual se reproducían las condiciones de vida y de trabajo de la clase obrera regional se vio profundamente modificado. La Reforma del Estado produjo una forma de Estado caracterizado - entre otros elementos- por la descentralización de las dependencias y funciones en los niveles locales y por la focalización de políticas públicas. De esta manera, los niveles estatales locales entran en relación directa con los organismos nacionales e internacionales de financiamiento de políticas públicas focalizadas, al mismo tiempo que la empresa privatizada articula intervenciones vinculadas con problemáticas sociales (salud, adicciones, contaminación, alimentación) y dirigidas a conjuntos sociales específicos (jóvenes, trabajadores desocupados, estudiantes, etc). En dichas articulaciones, las políticas de desarrollo local y de Responsabilidad Social Empresaria tienden a descargar sobre organizaciones de la sociedad civil la gestión de estos recursos. En este sentido, resulta relevante observar la forma en que, en el caso de San Nicolás de los Arroyos, el entramado relacional configurado en torno de las asociaciones vecinales y sindicales conserva el rol dominante como articulador de las prácticas de los trabajadores y de los trabajadores desocupados frente a la crisis social.

\section{A modo de cierre: rupturas, continuidades y nuevas preguntas}

Desde la instalación de la planta siderúrgica en sus inmediaciones, la dinámica de la ciudad de San Nicolás de los Arroyos se ha visto determinada por ella y se desplegó mediante un entramado de relaciones e instituciones que modelaron y fueron modeladas por las relaciones entre clases sociales a escala local. El entramado que reconstruimos se inscribe en el proceso hegemónico del nacionalismo industrialista, que supone un proyecto de acumulación traccionado por la inversión industrial, expresado por una forma de Estado que contempla tanto la intervención directa en la configuración de sectores productivos puntuales (estratégicos) como mecanismos de integración subordinada de la clase trabajadora a la reproducción del capital.

De una parte, las políticas empresarias de reclutamiento y segmentación de la fuerza de trabajo rápidamente contribuyeron a la configuración de un colectivo fabril fuertemente segmentado y jerarquizado y, a la vez, grupo obrero diferenciado del resto de los grupos obreros de la ciudad. En el ámbito extra- fabril, la segmentación entre los somiseros tomó

44 FIGARI Claudia y GINIGER Nuria, “iDe qué son responsables las empresas? La RSE en la consolidación de la hegemonía empresarial", ponencia presentada en VII Congreso de la Asociación Latinoamericana de Sociología del Trabajo, Sao Paulo, Brasil, Julio 2013. 
la forma de un company town restringido a los estratos superiores de la pirámide jerárquica, que, a su vez, remitía a la formación de un mercado de trabajo relativamente cerrado y especializado.$^{45}$ Estas políticas empresarias delimitan la investigación en torno de las políticas estatales respecto de los trabajadores como vía de construcción de elementos explicativos de la relación empresa- comunidad de emplazamiento. En este sentido, mostramos que, para el grueso de los trabajadores de la siderúrgica, los principales procesos que hacen a las condiciones de reproducción de la fuerza de trabajo fueron viabilizados mediante la organización sindical. ${ }^{46}$ Para la década del ' 70 el accionar sindical de los metalúrgicos había desbordado el espacio fabril para avanzar en reivindicaciones más amplias. El nivel municipal del Estado y las fuerzas sociales de anclaje local procesaron estas transformaciones con intervenciones que procuraban recuperar el control sobre los procesos desencadenados por la instalación de la empresa, sin lograr contrarrestar la dinámica que ésta imponía. Esta contradicción se tradujo en construcciones simbólicas que nutrieron el sentido común nicoleño y que hacían de los somiseros y sus organizaciones factores exógenos, disruptivos de la dinámica de la ciudad.

La crisis y transformación determinadas a nivel local por el proceso de privatización y reconversión productiva de la empresa siderúrgica, transformó sustancialmente las relaciones que sostenía aquel colectivo fabril singular con la comunidad. Este grupo obrero que gozaba de mejores condiciones relativas de reproducción y que dinamizaba el proceso de acumulación local se vio convertido en un contingente de trabajadores sometidos a la desocupación, la subocupación o la precarización laboral. Asimismo, las transformaciones en la forma de Estado - sintetizadas en el proceso de Reforma del Estado-supusieron la configuración de un conjunto de intervenciones focalizadas sobre estos conjuntos sociales. Los datos que hemos construido a lo largo del trabajo de campo, evidencian la forma en que estas políticas públicas focalizadas fueron vehiculizadas por el entramado institucional que había caracterizado el accionar obrero en la etapa anterior. En efecto, las organizaciones sindicales, las asociaciones vecinales (entre otras entidades culturales y sociales) fueron elementos centrales para la articulación de las políticas estatales y para continuar canalizando

45 En los abordajes sobre los paisajes industriales, el barrio -en tanto company town restringido"...es parte de la infraestructura que hace posible la producción" (nuestra traducción) PORTEUS J D "The nature of the company town", en Transactions of the Institute of British Geographers , Noviembre $1970, \mathrm{~N}^{\circ} 51$.

46 Esta situación presenta una diferencia importante respecto de las relaciones con la empresa presentes en las formas clásicas de company town, puesto que en el entramado institucional y la experiencia política y sindical de la clase obrera argentina ya había operado el pasaje hacia el idioma de los derechos en la construcción de reivindicaciones y demandas. GÓMEZ GALVARRIATO, Aurora, "From company towns to union towns: textile workers and the revolutionary state in Mexico", en DINIUS Oliver y Vergara Ángela (compiladores), Company Towns...,Op. Cit. y MORAES MOREL, Regina. A ferro e fogo..., Op. Cit. 
las prácticas de participación de los trabajadores en el marco de políticas de desarrollo local. En este sentido, la dualidad exógeno/local como forma predominante en que se explicaban las tensiones locales, se vio desplazada por la de emprendedores/SOMISA dependientes como síntesis de las nuevas marcas sobre la que gravitan las relaciones sociales: la capacidad de adaptación, la flexibilidad frente a contextos cambiantes, el ansia de progreso y el individualismo. En este contexto, tampoco resultan menores las intervenciones culturales, deportivas y en educación y salud, mediante las cuales la empresa privatizada se posiciona como un referente para la comunidad toda y ya no sólo para sus trabajadores.

Recibido: 22/07/2013

Aceptado: 26/09/2013 